\title{
Efecto de las palabras interrogativas en la inflexión final de enunciados en un corpus oral del mapudungun: estudio exploratorio ${ }^{1}$
}

Effect of interrogative words in sentence-final inflections of utterances from an oral corpus of Mapudungun: exploratory study

\section{Magaly Ruiz Mella \\ Universidad de La Frontera \\ Chile}

\author{
Mauricio A. Figueroa Candia \\ Universidad de Concepción \\ Chile
}

\section{Antonio Chihuaicura Chihuaicura}

Departamento de Administración de Educación Municipal - Tirúa Chile

\section{(c) $($ i $\ominus$}

Magaly Ruiz Mella: Departamento de Lengua, Literatura y Comunicación, Facultad de Educación, Cs. Sociales y Humanidades, Universidad de La Frontera, Chile. I E-mail: magaly.ruiz@ufrontera.cl

Mauricio A. Figueroa Candia: Departamento de Español, Facultad de Humanidades y Arte, Universidad de Concepción, Chile. I E-mail: maufigueroa@udec.cl

Antonio Chihuaicura Chihuaicura: Encargado Comunal Educación Parvularia DAEM, Tirúa, Chile.

| E-mail: achiwaikura@gmail.com 


\section{Resumen}

Este estudio exploratorio presenta los resultados cuantitativos sobre el efecto de las palabras interrogativas en la curva de tono de las dos últimas sílabas de enunciados interrogativos del mapudungun. La muestra analizada corresponde a 81 enunciados de pregunta emitidos por tres hablantes nativos representantes de tres zonas de la región de la Araucanía: Lonquimay, Cholchol y Padre las Casas. Los resultados indican que la mayoría de los enunciados interrogativos evaluados presentan ascensos entonativos finales; asimismo se aprecia una clara tendencia a que los enunciados sin palabra interrogativa manifiesten ascensos entonativos más pronunciados que los de los enunciados con palabra interrogativa. El contraste entre la presencia o ausencia de un término interrogativo en los valores de frecuencia fundamental de las preguntas en mapudungun es estadísticamente significativo en los enunciados del hablante de Lonquimay, pero no así en los enunciados de los hablantes de Cholchol y Padre las Casas. Estos resultados, que se discuten bajo la luz de relaciones de contacto lingüístico y de recursos pragmáticos para la transmisión de información interrogativa, sugieren que los enunciados interrogativos descendentes del castellano pueden estar ejerciendo una influencia en las variantes del mapudungun con las que tienen mayor contacto, y que ascensos entonativos finales relativamente grandes son el mecanismo vernáculo primario de transmisión de información pragmática interrogativa en mapudungun, excepto cuando las partículas interrogativas codifican esta información mediante foco estrecho.

Palabras clave: palabra interrogativa; inflexión final; mapudungun; corpus oral.

\section{Abstract}

This exploratory study provides quantitative evidence regarding the effect of interrogative words in sentence-final inflections of the last two syllables of interrogative utterances from Mapudungun. The sample contains 81 interrogative utterances spoken by three native speakers of Mapudungun, representing three different geographical areas from the Araucanía region: Lonquimay, Cholchol and Padre las Casas. Results showed that most interrogative utter-

1 Se hacen explícitos los agradecimientos al Proyecto Fondecyt de Iniciación №¹1150774 "Reflexión sobre el uso de los rasgos suprasegmentales, como el acento y la entonación, en la enseñanza de la lengua mapuche en la escuela”. 
ances display a sentence-final rise in fundamental frequency; also, there is a clear trend to find larger rises in utterances that do not have interrogative words. These differences were statistically significant in Lonquimay, but not in Cholchol or Padre las Casas. These results, which are discussed in relation to dynamics of linguistic contact and pragmatic resources to convey information about interrogative clauses, suggest that descending interrogative utterances from Chilean Spanish might be influencing those variants of Mapudungun in which there is more linguistic contact with Spanish, and that relatively large sentence-final rises are the primary vernacular mechanism to convey interrogative pragmatic information in Mapudungun, except when interrogative words codify this information via a narrow focus.

Keywords: interrogative words; sentence-final inflection; Mapudungun; oral corpus. 


\section{Introducción}

Las oraciones interrogativas, desde una perspectiva semántica, son expresiones abiertas que requieren que sean respondidas por el interlocutor. De acuerdo al tipo de respuesta que se solicita, las oraciones interrogativas se puede clasificar en: (a) interrogativa total o polar, cuyo propósito es que el interlocutor responda sí o no; (b) interrogativa alternativa, en cuya formulación está contenida la respuesta, y (c) alternativa parcial, que se caracteriza por ir encabezada por una palabra interrogativa (Di Tullio, 2005). También es posible clasificar las oraciones interrogativas desde un punto de vista gramatical en dos grupos: las preguntas con palabra interrogativa y las que no la contienen (Sosa, 1999). Esta última clasificación es la que se considerará en este estudio.

Desde un punto de vista prosódico, las oraciones interrogativas que contienen una palabra interrogativa concentran la intensidad fonética - entendida como un aumento de la intensidad, duración y/o frecuencia fundamental relativas - en ese componente de la oración, funcionando como foco estrecho. Este opera como una estrategia por parte del enunciador para intentar lograr centrar la atención del oyente en la palabra interrogativa, y se realiza prosódicamente con un ascenso en el constituyente interrogativo, lo que permite distinguir a estas oraciones de las declarativas (Di Tullio, 2005; Zúñiga, 2006). Asimismo, se afirma que estas oraciones tienen una intención cognoscitiva. Por esa razón, al menos en español, el término interrogativo es producido con el tono más alto de la curva tonal de la pregunta con el propósito de que el interlocutor formule su respuesta en concordancia con lo que pide el emisor, como se ilustra en el ejemplo dado por Sosa (1999: 147), proveniente de un sujeto de sexo femenino, hablante de una variante del español caribeño:

(1) ¿Dónde están los niños?

$H^{*} L^{*}+H \quad L^{*} L \%$

También es importante destacar que las preguntas con palabra interrogativa pueden manifestarse con ascenso o caída posnuclear, dependiendo de la urgencia de la respuesta. Si la pregunta (1) es realizada con un tono más bien neutro, las sílabas posnucleares pueden manifestarse con un ascenso (HH\%); en cambio, cuando la pregunta (1) es hecha de forma apremiante, las sílabas posnucleares suelen realizarse con un descenso del tono ( $\left.{ }^{*} L \%\right)$. Este fenómeno ha sido descrito para el español de Chile y otros dialectos del español (Prieto y Roseano, 2010).

Para el mapudungun, Smeets (2008) provee un listado de las palabras más frecuentemente utilizadas para realizar preguntas con un término interrogativo: iney (iniy, iñey), chem, chew, chumül, chumal, chumngelu, chumngechi, tuchi(chuchi), tunté-n (chunté-n)y mufü. Por ejemplo:

(2) ¿Chum amuy? (¿Cómo se fue?)

(3) ¿lñey amuy? (¿Quién fue?) 
Otro aspecto importante a destacar en la construcción gramatical de la lengua mapuche es lo que señala Zúñiga (2006), quien hace hincapié en las diferencias en la manera en la que se manifiestan y utilizan las partículas interrogativas entre el español y el mapudungun. Para la pregunta en español (4), las posibilidades de modificaciones que ofrece la lengua castellana son muy reducidas; en cambio, en el mapudungun se pueden registrar cuatro formas de preguntar lo mismo utilizando las partículas am, ama, anta, como se observa en los ejemplos tomados de Zúñiga (5-8):

(4) ¿Quién Ilegó?

(5) ¿lney akuy? ～(¿Quién Ilegó?)

(6) ¿lney am akuy? （¿Quién llegó [aquí]?)

(7) ¿lney akuy ama? (¿Quién Ilegó [recientemente]?)

(8) ¿lney anta akuy? (¿Quién llegó [la persona]?)

La pregunta (5) está construida con una estructura no canónica en la lengua mapuche, aunque es gramatical; esta forma sigue un patrón del español, es poco frecuente y puede encontrarse de forma aislada. En la pregunta (6) el emisor requiere específicamente saber quién llegó "aquí, a este lugar, para ello utiliza la partícula am. La forma interrogativa con la partícula ama (7) sugiere que la llegada de alguien es muy reciente; y en la última pregunta, con la partícula anta (8), el emisor demanda saber exactamente "quién” llegó al lugar. Sin embargo, el mismo autor advierte que no está claro el significado preciso de estas y otras partículas del mapudungun, ya que pueden ser encontradas en oraciones interrogativas y afirmativas con significados difíciles de precisar (Zúñiga, 2006: 258).

En relación con los rasgos suprasegmentales del mapudungun, las descripciones se concentran especialmente en el acento y la entonación, pero en su mayoría sin la utilización y medición de variables acústicas mediante instrumentos y software especializados. Croese (1980) provee algunos datos sobre al acento de las palabras en lengua mapuche, el cual, afirma, puede recaer en cualquiera de las sílabas de un término; no obstante, otros autores indican que generalmente, en un enunciado, la penúltima y última sílaba son las acentuadas (Smeets, 2008; Zúñiga, 2006).

En cuanto a la entonación de la lengua mapuche, Catrileo (2010) afirma que el patrón nuclear ascendente o descendente de la curva melódica dependerá de dos factores: (a) el área dialectal y (b) la intención de cada hablante. Al respecto, la autora describe la culminación posnuclear con ascensos y descensos tanto para las oraciones interrogativas como las declarativas.

Precisamente, una de las dificultades en el estudio de la entonación del mapudungun consiste en la falta de patrones sistemáticos en las terminaciones de los enunciados declarativos 
e interrogativos, dado que se observa alta variabilidad dentro de cada hablante y entre ellos. Los pehuenches (provenientes de la zona de Lonquimay) realizan oraciones declarativas con tonos ascendentes, similares a los registrados para los hablantes de Chiloé (Muñoz-Builes, Ramos, Román, Quezada, Ortiz-Lira, Ruiz y Atria, 2017). Esta característica, en cambio, es menos frecuente en los hablantes mapuches de las zonas del valle como es el caso de Padre las Casas y Cholchol (Ruiz, Ulloa y Chihuaicura, 2019).

En este primer acercamiento al estudio acústico de las oraciones interrogativas del mapudungun, se observará el comportamiento del ascenso tonal de las dos últimas sílabas de oraciones interrogativas con y sin palabra interrogativa, emitidas por tres hablantes nativos de tres zonas de la Región de la Araucanía, con la finalidad de realizar un aporte al conocimiento de las características fonético-acústicas de la entonación de esta lengua vernácula, mediante herramientas cuantitativas.

\section{Sección de métodos}

\subsection{Características de los participantes}

El corpus de enunciados interrogativos obtenido para este estudio proviene de entrevistas semidirigidas que tres hablantes nativos de mapudungun — dos hombres y una mujer - realizaron a hablantes nativos de la misma lengua de las comunidades en las que los entrevistadores residen: Lonquimay, Cholchol y Padre las Casas, todas ubicadas en la Región de la Araucanía (las grabaciones analizadas aquí son del habla de los entrevistadores, no de los entrevistados). Los tres sujetos tenían el rol de facilitador de cada entrevista. Todos los entrevistadores adquirieron el mapudungun en su infancia, eran adultos jóvenes entre 25 y 35 años de edad, con educación formal completa, y continuaban viviendo en sus comunidades, lo que les permitió lograr el dominio de la lengua por el ejercicio continuo con los hablantes ancianos monolingües del mapuche.

\subsection{Tarea de elicitación}

La conversación semidirigida entre el entrevistador y cada participante se dividió en tres momentos. En la primera etapa, trató sobre saludos tradicionales, descripción general de la vida del entrevistado, su oficio, aspectos familiares y otros datos personales. En la segunda etapa, el entrevistador realizaba preguntas relacionadas con algún problema de la comunidad, con la finalidad de obtener textos orales argumentativos; por ejemplo, sobre la falta de agua por la desforestación o sobre la enseñanza del mapudungun en la escuela. De esta segunda etapa se extrajeron los enunciados interrogativos. Por último, en la tercera etapa, los participantes debían narrar un momento de sus vidas en el que hubiesen estado en peligro de muerte él o alguno de sus seres queridos. Toda la interacción comunicativa se llevó a cabo en lengua mapuche, resultando en un promedio de 30 minutos de grabación por entrevista. La tabla 
1 expone el detalle de entrevistas según zonas estudiadas y cantidad de interacciones por sujeto. Como se aprecia, solamente el entrevistador de Cholchol realizó tomas de datos fuera de su propia comunidad.

\section{TABLA 1}

Cantidad de entrevistas en cada zona realizada por los tres entrevistadores

\begin{tabular}{lcccc} 
ENTREVISTADOR & LONQUIMAY & CHOLCHOL & PADRE LAS CASAS & TOTAL \\
$\begin{array}{l}\text { Entrevistador } 1 \\
\text { Lonquimay }\end{array}$ & 7 & 0 & 0 & 7 \\
\hline $\begin{array}{l}\text { Entrevistador } 2 \\
\text { Cholchol }\end{array}$ & 4 & 4 & 3 & 11 \\
\hline $\begin{array}{l}\text { Entrevistador } 3 \\
\text { Padre las Casas }\end{array}$ & 0 & 0 & 3 & 3 \\
\hline
\end{tabular}

\subsection{Condiciones de grabación, equipamiento utilizado y evaluación de las señales}

Las grabaciones se llevaron a cabo en cada comunidad estudiada, en el hogar de los entrevistados, en la habitación que estuviese más aislada acústicamente. Para registrar los enunciados, se utilizó una grabadora TASCAM DR-22WL. Esta grabadora posee dos micrófonos cardioides de condensador en formato $X-Y$, lo que permite grabar al mismo tiempo el habla del entrevistador y del entrevistado. Los entrevistadores ubicaron este dispositivo entre ellos mismos y los sujetos, teniendo la precaución de no situar los micrófonos más lejos que 30 centímetros del rostro de los participantes de la entrevista. Todas las entrevistas fueron grabadas en formato digital WAV, mono (el registro de ambos micrófonos alimentaba una sola señal), con una frecuencia de muestreo de $44.100 \mathrm{~Hz}$ y una profundidad de 16 bits. Dado que las condiciones de grabación no fueron siempre las óptimas, se llevó a cabo una evaluación formal de la relación señal-ruido de las grabaciones, la cual mostró ser suficiente para el desarrollo de un estudio de frecuencia fundamental ( $\bar{x}=24,334 \mathrm{~dB}, \sigma=6,314 \mathrm{~dB}$ ).

\subsection{Anotación, procesamiento de señales y extracción de datos}

Los enunciados recogidos fueron etiquetados y segmentados en TextGrids del programa Praat (Boersma y Weenink, 2018). Para cada audio, se transliteró en mapudungun el contenido del enunciado, se segmentaron y etiquetaron las sílabas, y se identificó el punto medio de cada vocal. Se procedió luego a crear un objeto Pitch de Praat basado en la señal de audio, mediante el método de doble paso (De Looze y Hirst, 2008), que genera una estimación de la curva de frecuencia fundamental mejor adaptada a las características vocales de cada sujeto, a diferencia de otros métodos que utilizan valores por defecto. Cada uno de los objetos Pitch fue revisado manualmente, con la finalidad de corregir potenciales errores de estimación de 
la curva de tono que todavía pudiesen estar presentes, como saltos octavales o aparición de información de $f_{0}$ en zonas silentes. Finalmente, se utilizó la información temporal registrada en los TextGrid y el objeto Pitch para obtener los valores de frecuencia fundamental del punto central de las dos últimas vocales, y se procedió a calcular su diferencia restando el valor de la penúltima vocal al de la última (Muñoz-Builes, Ramos, Román, Quezada, Ortiz-Lira, Ruiz y Atria, 2017), de manera tal que una diferencia positiva significa que hubo un ascenso en la sección final de la curva de tono, una diferencia negativa da cuenta de un descenso en la curva de tono y una diferencia de cero significa que no hubo movimiento de frecuencia fundamental entre esos dos puntos (véase la figura I para una ilustración de este procedimiento). Todos los valores de frecuencia fundamental obtenidos fueron medidos en semitonos (nivel de referencia: $100 \mathrm{~Hz}$ ), con la finalidad de poder comparar los datos obtenidos de los distintos sujetos.

\section{FIGURA 1}

Representación de la forma de onda, espectrograma, curva de frecuencia fundamental y procedimientos de etiquetado y segmentado de un enunciado perteneciente a la entrevistadora de Padre las Casas. La escala del espectrograma se extiende de o a $5.500 \mathrm{~Hz}$, y la de la curva de $f_{0}$, de 0 a $500 \mathrm{~Hz}$. Se han destacado con barras verticales más gruesas las posiciones centrales de la penúltima y última vocal; para cada enunciado, se ha restado el valor de la penúltima vocal medido en semitonos (aquí, "v7") al de la última (aquí "v8”), lo que en este caso resulta en un valor positivo que da cuenta de un ascenso final en la curva de tono.

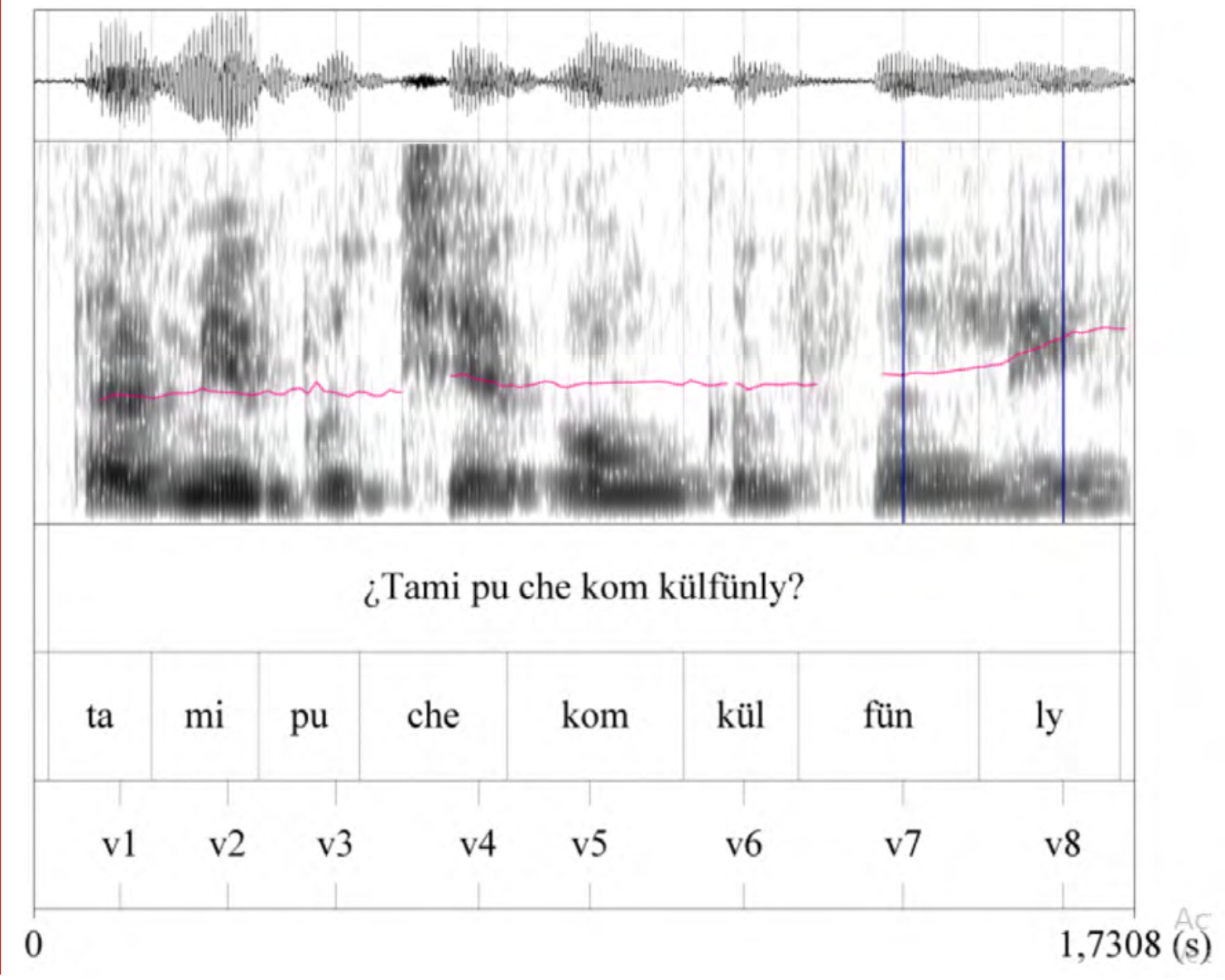




\section{Sección de resultados}

\subsection{Características del corpus}

Los datos provenientes del corpus, constituido por 89 enunciados, fueron importados al programa $R$ (R Core Team, 2018), en el que se realizaron todos los subsecuentes análisis. Una inspección preliminar de los datos reveló la presencia de algunos valores atípicos para las distribuciones de diferencias de semitonos de la muestra, los cuales fueron removidos mediante el criterio de 2.5 distancias de desviación absoluta respecto de la media, considerado como un procedimiento conservador en la bibliografía especializada (Leys, Ley, Klein, Bernard y Licata, 2013). En total, 8 enunciados fueron eliminados, de manera que el corpus se redujo a 81 enunciados, cuyo resumen se presenta en la tabla 2.

\section{TABLA 2}

Distribución de los enunciados interrogativos por zona y presencia de palabra interrogativa

\begin{tabular}{lccc} 
ZONAS & $\begin{array}{c}\text { CON PALABRA } \\
\text { INTERROGATIVA }\end{array}$ & $\begin{array}{c}\text { SIN PALABRA } \\
\text { INTERROGATIVA }\end{array}$ & TOTAL \\
Lonquimay & 13 & 17 & 30 \\
\hline Cholchol & 12 & 8 & 20 \\
\hline Padre las Casas & 15 & 16 & 31 \\
\hline
\end{tabular}

\subsection{Resultados y análisis estadísticos}

Una síntesis de los datos de diferencias de frecuencia fundamental se presenta en la figura 2. Como puede ser observado, la mayoría de los enunciados interrogativos evaluados presenta ascensos entonativos finales, puesto que relativamente pocos de ellos se encuentran ubicados bajo el valor cero del eje vertical. Para las tres zonas geográficas se aprecia también una clara tendencia a que los enunciados sin palabra interrogativa presenten ascensos entonativos más pronunciados que los de los enunciados con palabra interrogativa. Con respecto a la variable zona geográfica, se observa una mayor dispersión de valores para la zona de Cholchol y una tendencia de Lonquimay a presentar ascensos más pronunciados.

Con la finalidad de evaluar si las tendencias observadas tienen sustento estadístico, se llevó a cabo una prueba ANOVA de dos vías que evaluó el efecto de las variables independientes zona geográfica y presencia de palabra interrogativa en los valores de la variable dependiente diferencia de frecuencia fundamental. Los resultados del análisis revelaron que no existe una interacción estadísticamente significativa entre las variables evaluadas, $F(2,75)=0,331, p=0,719$, es decir, ninguna de las variables independientes modula a la otra en la expresión de la variable dependiente, pero sí se detectó un efecto principal de la variable zona geográfica, $F(2,75)=$ $3,163, p<0,05$, y para la variable presencia de palabra interrogativa, $F(1,75)=5,042, p<0,05$, lo que 
confirma las tendencias descritas más arriba. Además de la prueba ANOVA, se llevaron a cabo pruebas $t$ para determinar si las diferencias observadas entre los enunciados con y sin palabra interrogativa, dentro de cada zona geográfica, son estadísticamente significativas. En el caso de los datos de Lonquimay, los valores de los enunciados con palabra interrogativa $(\bar{x}=2, \sigma=$ $1,83)$ y los de los enunciados sin palabra interrogativa $(\bar{x}=3,6, \sigma=1,84)$ presentaron diferencias estadísticamente significativas, $t(26,047)=-2,382, p<0,05$. En el caso de los datos de Cholchol, Ios enunciados con palabra interrogativa $(\bar{x}=1,18, \sigma=3,01)$ y los enunciados sin palabra interrogativa $(\bar{x}=1,93, \sigma=4,04)$ no presentaron diferencias estadísticamente significativas, $t(12,08)=$ $-0,446, p=0,332$. Algo similar ocurre con los datos de Padre las Casas, en los que los enunciados con palabra interrogativa $(\bar{x}=1,07, \sigma=1,43)$ y los enunciados sin palabra interrogativa $(\bar{x}=1,79$, $\sigma=2,16)$ no presentan diferencias estadísticamente significativas, $t(26,195)=-1,095, p=0,142$.

\section{FIGURA 2}

Gráficos de caja para los datos de diferencias de frecuencia fundamental organizados por las variables zona geográfica y presencia de palabra interrogativa. La línea horizontal dentro de cada caja indica la posición de la media de cada distribución; la zona superior, inferiory cada cola de cada caja contienen un cuartil de la distribución. Los valores negativos de la escala vertical corresponden a descensos de la curva de tono, mientras que los valores positivos, a curvas ascendentes. La distancia de los valores con respecto al cero indica la magnitud de la diferencia de tono: a mayor distancia del cero, mayor diferencia entre los valores medidos en la penúltima y última vocal.

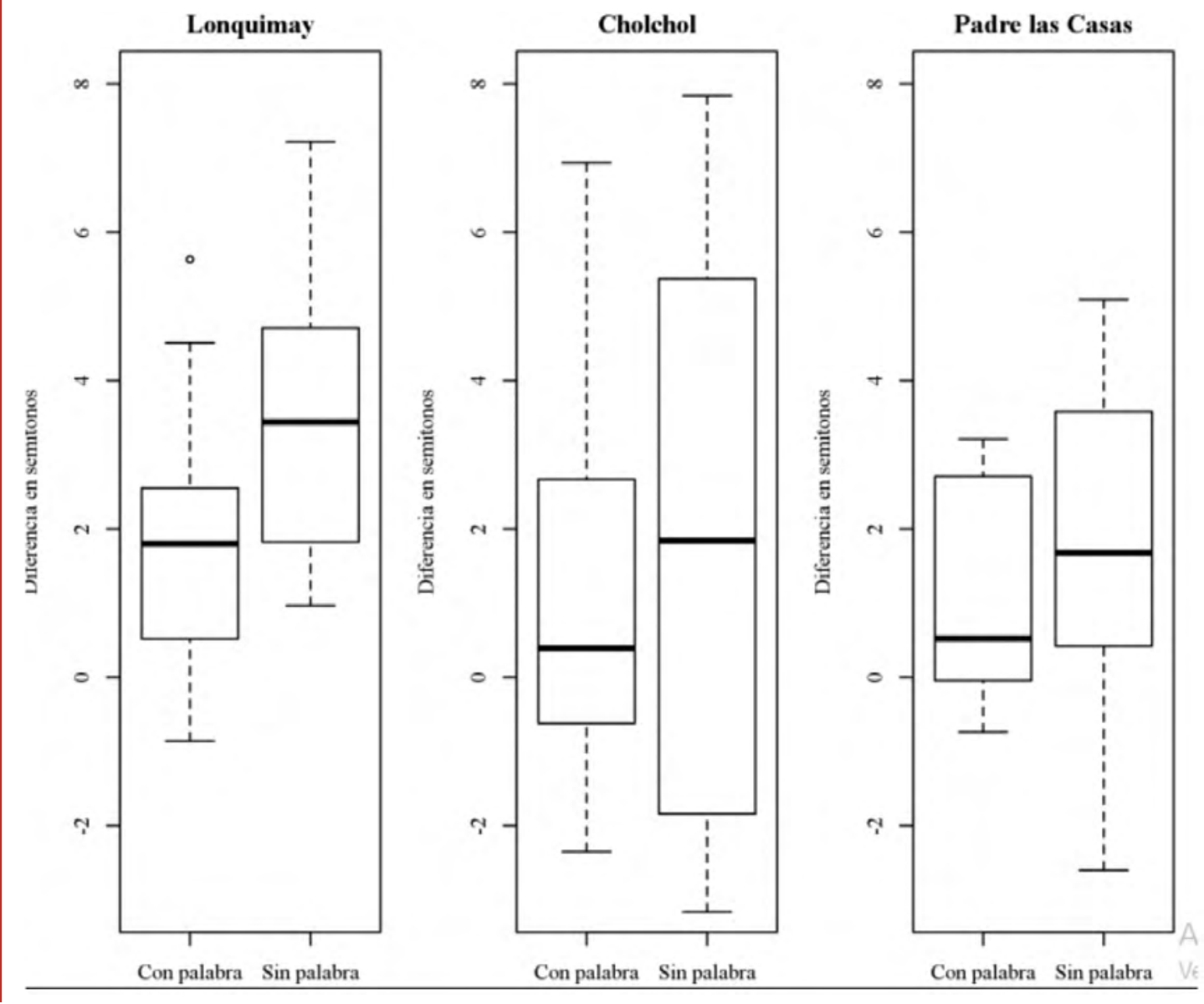




\section{Discusión}

La discusión de los resultados se organizará en torno a dos factores que han demostrado tener un efecto en el comportamiento melódico de las construcciones interrogativas: el área geográfica y dialectal de los hablantes y la intención comunicativa en el contexto de entrevista semiestructurada.

En cuanto al primer factor, es un hecho conocido que existen diferencias dialectales según la zona geográfica en donde se asiente la comunidad de hablantes mapuches. De acuerdo a los estudios de Croese (1980), quien divide geográficamente a la población mapuche en 8 subgrupos, y según el criterio de identidad territorial mencionada en el Perfil Sociolingüístico (Gündermann, Canihuan, Castillo y Clavería, 2008), la población mapuche ubicada en el sector altoandino de las comunas de Alto Biobío y Lonquimay se denomina pehuenche, y pertenece al subgrupo IV de Croese. Al noroeste de Temuco, se ubican los nagches, mapuches del secano interior de Malleco, que incluye las comunas de Galvarino y Cholchol en la Provincia de Cautín. A solo 5 km al sur de la capital de la Región de la Araucanía, se ubican los huenteches, mapuches del sector de Padre las Casas, Temuco rural y Lautaro. Tanto nagches como huenteches pertenecen al subgrupo III, según el ordenamiento dialectal de Croese. En relación con la competencia lingüística de los hablantes de mapudungun de La Araucanía, el Perfil Sociolingüístico (Gündermann, Canihuan, Castillo y Clavería, 2008), según identidad territorial, registra el porcentaje más alto para la población pehuenche $(67,6 \%)$ en comparación con la población nagche (48\%) y huenteche (26,8\%). Esto confirma lo dicho por Salas, en torno a que "a mayor distancia de los centros urbanos es menor la densidad de contacto con hispano-hablantes, y en consecuencia, hay predominio del mapudungu sobre el castellano" (Salas, 1992: 35). Croese, por su parte, afirma que, mientras más cercanas sean las zonas dialectales, mayor será el grado de inteligibilidad entre ellas (1980). En este sentido, es esperable que los hablantes de Cholchol y Padre las Casas manifiesten un comportamiento similar en la emisión de enunciados interrogativos en mapudungun, y que ambos muestren algún grado de diferencia con el hablante de Lonquimay.

De acuerdo con los resultados de esta investigación, los tres hablantes presentan ascensos entonativos finales. Asimismo, manifiestan una clara tendencia a realizar los ascensos tonales más pronunciados en preguntas que no presentan palabra interrogativa. Sin embargo, el hablante pehuenche (de Lonquimay) registra alturas tonales en la inflexión final de mayor magnitud que las emitidas por los hablantes nagche y huenteche. Mientras los hablantes monolingües de mapudungun de Lonquimay se encuentran alejados de las zonas urbanas de la Región de la Araucanía, los hablantes de Cholchol y sobre todo Padre las Casas se encuentran en zonas cercanas o que colindan con la ciudad de Temuco y que tienen una relación comercial más estrecha con los hablantes de castellano residentes de esta ciudad. Si consideramos la afirmación de Salas, podría postularse que el hablante de Lonquimay manifiesta una variante "más mapuche" al encontrarse tan alejado de los centros urbanos de habla 
castellana, a diferencia del hablante nagche y sobre todo del hablante huenteche, quienes, potencialmente, pueden estar recibiendo mayor influencia del castellano y presentar una mayor influencia en los rasgos suprasegmentales.

Con respecto a la entonación, la tradición bibliográfica, partiendo desde Navarro Tomás (1944), ha descrito la entonación de las interrogativas del castellano como enunciados que pueden terminar en ascenso tonal. Quilis (1993) analiza once tipos de preguntas, entre ellas las interrogativas pronominales, o de palabra interrogativa, que caracteriza como realizaciones con un tono medio seguido de un tono bajo; interrogativas pronominales enfáticas con el tono más elevado, finalizando en un tono bajo, y las interrogativas con matiz de cortesía con el patrón con un tono final ascendente. Los autores que han descrito la melodía de las interrogativas del castellano coinciden en tres puntos: (a) las interrogativas presentan una melodía con tono alto a lo largo de toda la curva, culminando en una inflexión final ascendente, a diferencia de las declarativas; (b) las preguntas sin palabra interrogativa suelen terminar con un tono ascendente; y (c) las preguntas con palabra interrogativa (pronominales) no marcadas tienen una inflexión final descendente, a excepción de las preguntas con un tono cortés (Cid Uribe, OrtizLira y Valenzuela, 1998). Este último punto es importante: en el español, incluido el chileno, es posible terminar enunciados interrogativos con palabra interrogativa con inflexión final descendente. Reforzando lo anterior, desde un marco teórico de la fonología métrica autosegmental, se consigna la alternancia entre un ascenso tonal con una configuración nuclear de L*HH\%, quizás más esperable, y una inflexión final con un tono bajo L*L\% (Ortiz, Fuentes y Astruc, 2010). En cuanto a la entonación de variedades dialectales del sur de Chile, más cercanas a la muestra que se estudia aquí, Muñoz-Builes, Ramos, Román, Quezada, Ortiz-Lira, Ruiz y Atria (2017) analizaron el fenómeno del habla ascendente registrada en Chiloé. Este estudio acústico se concentró en el movimiento de $f_{0}$ en las dos últimas vocales de enunciados declarativos. Sus resultados mostraron un consistente y progresivo aumento de $f_{0}$ en oraciones declarativas, las cuales tendían a finalizar con una inflexión ascendente, un patrón entonativo que es similar al de los enunciados interrogativos. Este rasgo dialectal del habla sureña ascendente es una característica tonal que se extiende a otras zonas cercanas, entre ellas, la Región de la Araucanía.

El castellano y el mapudungun han convivido en Chile, especialmente en las regiones del Biobío y La Araucanía, por varios siglos. Este hecho puede contribuir a explicar por qué en zonas geográficas en las que se habla un mapudungun en mayor contacto con el español (Cholchol y Padre las Casas), las entonaciones ascendentes finales de enunciados con partícula interrogativa no sean tan pronunciadas como las de otras zonas, en las que el contacto es menor (Lonquimay), como lo explican mecanismos descritos en la bibliografía de contacto lingüístico (e. g., Silverstein, 1996; Winford, 2003). Si bien, como fuera mencionado antes, los enunciados interrogativos de Lonquimay tienen cierto parecido al habla ascendente de las declarativas de Chiloé, dado que en ambos casos predominan las inflexiones finales ascendentes, no resulta plausible sugerir una influencia de esta variedad dialectal del castellano chileno en el mapudungun de la zona pehuenche. 
En relación con el segundo factor referido a la intención comunicativa del hablante, se hace necesario hacer referencia de manera breve a la función informativa de foco. Esta es una función pragmática que aumenta la densidad informativa de ciertos constituyentes de un enunciado (Pinuer, 2009). Habitualmente, se materializa mediante mecanismos suprasegmentales; sin embargo, también existen otros recursos lingüísticos para focalizar, entre ellos el uso de partículas que marcan el componente de foco en el resto de la cláusula (Dik, 1997: 327). Por ejemplo, en las preguntas con palabra interrogativa del castellano, como las que incluyen "qué”, "dónde” y “cómo”, es esperable que el hablante destaque este componente por sobre otros constituyentes en la interacción comunicativa (realiza foco estrecho). Ahora bien, aun cuando haya focalización debido a la presencia de una partícula interrogativa, también es posible que rasgos suprasegmentales como la inflexión final complementen la intención pragmática del hablante.

Los resultados del presente estudio muestran que, en todas las zonas, y con independencia de la presencia o no de partículas interrogativas, existe información pragmática sobre la naturaleza interrogativa de los enunciados que se encuentra codificada mediante inflexiones finales ascendentes. Sin embargo, también es claro que la presencia de partículas interrogativas del mapudungun - las cuales, hipotetizamos, facultan un foco estrecho- atenúa este efecto, puesto que, en todas las zonas, los enunciados interrogativos con partícula interrogativa presentan inflexiones finales ascendentes de menor magnitud que aquellos enunciados que no las contienen. Con respecto a las diferencias entre las zonas geográficas, observadas en la figura 2, es posible que exista una tensión, en las zonas de mayor contacto con el español, esto es, Cholchol y Padre las Casas, entre la necesidad de focalizar el elemento interrogativo en los enunciados sin partículas interrogativas a través de inflexiones finales muy ascendentes (las cuales no se observan) y la influencia de enunciados interrogativos con inflexiones finales descendentes del castellano chileno. Esta potencial influencia de las interrogativas descendentes chilenas no se evidencia en Lonquimay, lugar en el que los ascensos de las interrogativas sin partícula son significativamente más altos que los de los enunciados interrogativos con partícula, dado su relativo aislamiento. Esto permite hipotetizar que los mecanismos originales de transmisión de información pragmática interrogativa del mapudungun, en enunciados sin partícula, son los ascensos finales. Debe tenerse en cuenta, sin embargo, que los alcances de la presente investigación exploratoria no permiten resolver esta y otras hipótesis planteadas anteriormente de manera concluyente.

\section{Conclusiones y proyecciones}

El presente estudio se propuso medir acústicamente los ascensos tonales de las dos últimas sílabas de enunciados interrogativos, con y sin partícula interrogativa, en tres variedades dialectales del mapudungun de la Región de la Araucanía. El análisis de los resultados y su discusión permiten concluir que: (a) los enunciados interrogativos del mapudungun, en gene- 
ral, manifiestan inflexiones finales ascendentes; (b) la ausencia de partículas interrogativas amplifica la magnitud de los ascensos finales; (c) en zonas dialectales del mapudungun con mayor contacto con el castellano los ascensos finales de enunciados sin partícula son de menor magnitud, potencialmente por influencia de enunciados interrogativos descendentes del castellano; esto también sugiere que los mecanismos vernáculos de transmisión de información pragmática interrogativa son los ascensos finales pronunciados, excepto cuando partículas interrogativas codifican la información pragmática sobre la interrogación mediante un foco estrecho. En cuanto a la metodología, como se ha demostrado aquí, la obtención de mediciones sobre movimientos entonativos en las dos últimas sílabas en mapudungun parece proveer información relevante sobre características suprasegmentales y pragmáticas de la lengua, lo que también permitirá realizar comparaciones entre variedades dialectales de la lengua mapuche.

Con respecto a las proyecciones, en futuras incursiones sobre temas similares, sería deseable contar con un mayor número de participantes, para confirmar o refutar de manera más definitiva las tendencias observadas acá. También, sería conveniente investigar otras zonas dialectales del mapudungun, como las zonas lafkenche (de la costa) y huilliche (del sur), que han demostrado ser relativamente distintas a las demás zonas dialectales (Croese, 1980; Salas, 1992). Finalmente, es necesario complementar estudios de producción como este con otros de carácter perceptivo, con la finalidad de determinar qué claves acústicas son las necesarias para que hablantes nativos de la lengua mapuche identifiquen enunciados como interrogativos.

\section{Bibliografía citada}

Boersma, Paul, y David WeEnINK, 2018: Praat: doing phonetics by computer [programa computacional]. Versión 6.0.39, descargada el 3 de abril de 2018 de http://www.praat.org/

Catrileo, María, 2010: La lengua mapuche en el siglo XXI, Valdivia: Universidad Austral de Chile.

Cid Uribe, Miriam, Héctor Ortiz-LiRa y Eugenia SaAvedra Valenzuela, 1998: “La entonación del enunciado interrogativo indagativo del español culto de Santiago de Chile", Boletín de Filología 37 (1), 355-378.

Croese, Robert A., 1980: “Estudio dialectológico del mapuche”, Estudios Filológicos 15, 7-38.

De Looze, Céline, y Daniel J. HIRST, 2008: "Detecting changes in key and range for the automatic modelling and coding of intonation”, comunicación presentada en Speech Prosody 2008 (eds.: Plínio A. Barbosa, Sandra Madureira y Cesar Reis), 135-138.

Dı Tulııo, Ángela, 2005: Manual de gramática del español, Buenos Aires: La Isla de la Luna. 
Dık, Simon D., 1997: The Theory of Functional Grammar. Part 1: The Structure of The Clause, Berlín y Nueva York: Mouton de Gruyter.

Gündermann, Hans, Jaqueline Canihuan, Ernesto Castillo y Alejandro Clavería, 2008: Perfil sociolingüístico de comunidades mapuche de la VIII, IX y X Región, Santiago, Chile: Universidad Tecnológica Metropolitana.

Leys, Christophe, Christophe Ley, Olivier Klein, Philippe Bernard y Laurent Licata, 2013: “Detecting outliers: Do not use standard deviation around the mean, use absolute deviation around the median”, Journal of Experimental Social Psychology 49 (4), 764-766.

Muñoz-Builes, Diana, Dania Ramos, Domingo Roman, Camilo Quezada, Héctor Ortiz-Lira, Magaly Ruiz y José Joaquín AtriA, 2017: “El habla ascendente de Chiloé: primera aproximación”, Onomázein 37, 1-15.

Navarro Tomas, Tomás, 1944: Manual de entonación española, New York: Spanish Institute in the United States.

Ortiz, Héctor, Marcela Fuentes y Lluïsa Astruc, 2010: "Chilean Spanish intonation" en Pilar Prieto y Paolo Roseano (eds.): Transcription of Intonation of the Spanish Language, München: Lincom Europe, 255-283.

Pinuer, Claudio, 2009: "La dimensión de focalidad: Conceptualización, instanciación y taxonomías", Revista Signos 42 (69), 83-106.

Prieto, Pilar, y Paolo Roseano, 2010: Transcription of intonation of the Spanish language, Munich: Lincom Europa.

Quilıs, Antonio, 1993: Tratado de fonología y fonética españolas, Madrid: Gredos.

R CORE TEAM, 2018: R: A language and environment for statistical computing [programa computacional]. Versión 3.4.4, descargada el 5 de abril de 2018 de https://www.R-project.org/

Ruiz, Magaly, Olga Ulloa y Antonio Chinuaicura, 2019: "Acento y Entonación en enunciados declarativos del español de Chile y mapudungun: primer acercamiento a la prosodia de ambas lenguas en contacto", Alpha 49, 299-314.

SalAS, Adalberto, 1992: El mapuche o araucano: fonología, gramática y antología de cuentos, Madrid: MAPFRE.

Silverstein, Michael, 1996: "Dynamics of linguistic contact", Handbook of North American Indians 17, 117-36. 
SmeEts, Ineke, 2008: A grammar of Mapuche, Berlín y Nueva York: Walter de Gruyter Mouton.

SosA, Juan Manuel, 1999: La entonación del español: su estructura fónica, variabilidad y dialectología, Madrid: Cátedra.

Winford, Donald, 2003: An introduction to contact linguistics, Oxford: Wiley-Blackwell.

Zúñiga, Fernando, 2006: Mapudungun, el habla mapuche, Santiago, Chile: Centro de Estudios Públicos. 